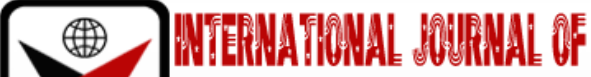

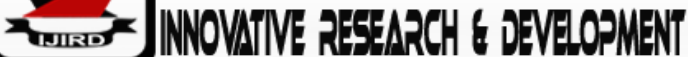

ISSN 2278-0211 (Online)

\section{Complex Frequency Domain Model Reduction: A Comparison of Techniques}

\author{
Sengchu Chow \\ Senior Lecturer, INTI International College, Malaysia
}

\begin{abstract}
:
A mathematical model of the plant to be controlled must first be identified in order to design a controller to improve the process' performance and stability. This mathematical model usually is in the form of complex frequency domain statespace realization or continuous/ discrete time domain regression algorithm. This paper works on the complex frequency domain state-space realization.

Reducing the order of the state-space model of the physical plant is usually desirable since it reduces computational time and hardware implementation for the resulting controller designed based on the model.

For a frequency domain state space realization of a linear time-invariant and non-causal plant, there are numerous methods available to reduce its order. These include Balancing Model Reduction, Frequency-Weighted Balancing, LowFrequency Approximation and Balanced Truncation with Reduced Error Bound.

Besides background information and a summary, the model-reduction methodology for each of the above-said techniques is also presented.

Using a two-input two-output six-state model for a large turbo-generator and a large industrial boiler with 8 states, 2 inputs and 2 outputs model, this paper provides a comparison between three of the four methodologies mentioned above in terms of the spectral norms of the model reduction error.
\end{abstract}

Keywords: Reduced order modeling, model reduction error, balanced truncation with reduced error bound, spectra norm of error

\section{Introduction}

The order of a given plant's mathematical model depends on the number of states necessary to fully describe the plant's internal operation. However, a lower order controller for a high order plant is often desirable for reasons of requiring fewer components in hardware implementation, reduced computational time and achieving higher controller reliability.

Several techniques are available in the literature for deriving reduced-order models and lower order controllers. Among various model-reduction techniques in both frequency and time domains, balanced-truncation method has many advantages, including the guarantee of a stable and minimal reduced-order model for a given stable and minimal system. However, the frequency domain characteristics of these reduced-order models are such that the spectral norm of the model reduction error is large at low frequencies and small at high frequencies. Liu [7] and Prakash [10] have introduced Singular Perturbation Approximation to balanced systems that offers a good match between the reduced-order model and the origin model at low frequencies. In this method, the model reduction error is small at low frequencies and approaches a finite value at high frequencies.

By introducing a weighting factor, a modified balanced-truncation reduced-order model was developed by Chow [1]. This weighting factor provides an extra degree of freedom in emphasizing the relative magnitude of the model reduction errors at low and high frequencies. The direct method of Moore [9] and the singular perturbation approximation introduced by Prakash [10] became two special cases in the modified technique. The maximum model reduction error can be reduced to a lower value compared to [9] and [10] by an appropriate choice of the weighting factor.

\section{Review of Literature}

\subsection{Common Nomenclature}

Let a balanced state-space realization of a system $\mathrm{G}(\mathrm{s})$ be

$$
G(s)=(A, B, C, D)=\left[\begin{array}{ll}
A & B \\
C & D
\end{array}\right] \mid
$$

with controllability/observability gramian giveh by

$$
\sum=\operatorname{diag}\left\{\sigma_{i}\right\}=\left[\begin{array}{cc}
\sum_{1} & 0 \\
0 & \sum_{2}
\end{array}\right]
$$


in which

$$
\sigma_{1} \geq \sigma_{2} \geq \cdots \geq \sigma_{n} \quad \text { and } \quad \sigma_{r} \gg \sigma_{r+1}
$$

where $\sum_{1} \in R^{r \times r}, \sum_{2} \in R^{(n-r) \times(n-r)}, r<n$ and $n$ is the order of the system. If the system has $m$ inputs and $p$ outputs, then the dimensions of the four matrices are $A_{n \times n}, B_{n \times m}, C_{p \times n}$ and $D_{p \times m}$.

The matrices $A, B$ and Care partitioned compatibly with $\sum$ as

$$
A=\left[\begin{array}{ll}
A_{11} & A_{12} \\
A_{21} & A_{22}
\end{array}\right], B=\left[\begin{array}{l}
B_{1} \\
B_{2}
\end{array}\right] \text { and } C=\left[\begin{array}{ll}
C_{1} & C_{2}
\end{array}\right] \text {. }
$$

\subsection{Balancing Model Reduction}

This is a direct truncation method proposed by Moore [9] in which the weaker subsystem $A_{22}$ is boldly discarded leaving the reduced-order model as

$G_{r}(s)=\left(A_{11}, B_{1}, C_{1}, D\right)$.

The upper bound for the model reduction error introduced by this truncation is proved by Enns [2] to be

$E_{\infty} \equiv\left\|G(s)-G_{r}(s)\right\|_{\infty} \leq 2 \operatorname{tr}\left[\sum_{2}\right]=\sum_{i=r+1}^{n} \sigma_{i}$.

The motivation for the use of the $L_{\infty}$-norm error criterion (as oppose to the classical integral square of some function) is that it is the magnitude of the highest peak of the error function over frequency that is important due to stability requirement, as proposed by Ridgely [11].

\subsection{Low Frequency Approximation}

It was discovered by Prakash and Rao [10] that the method proposed in Section 2.2 above introduces a model reduction error which is large in low frequencies and small in high frequencies. This is not desirable for control applications since most practical systems do operate in low frequency ranges. Prakash and Rao proposed a reduction technique which offers a good match at low frequencies while allowing the reduction error to be higher at high frequencies. This method is summarized below.

Based on the nomenclature presented in section 2.1, a reduced-order model for $G$ is obtained as

$G_{r}(s)=\left(A_{r}, B_{r}, C_{r}, D_{r}\right)$

where

$$
\begin{aligned}
& A_{r} \equiv A_{11}-A_{12} A_{22}^{-1} A_{21} \\
& B_{r} \equiv B_{1}-A_{12} A_{22}^{-1} B_{2} \\
& C_{r} \equiv C_{1}-C_{2} A_{22}^{-1} A_{21} \\
& D_{r} \equiv D-C_{2} A_{22}^{-1} B_{2} .
\end{aligned}
$$

It has been proven that the reduction error equals to zero at zero frequency and approach a constant finite value as the frequency tends to infinity. Also, this reduced order model is guaranteed to be stable and minimal. The model reduction error bound is the same as the one given in (2.6).

\subsection{Frequency-Weighted Balancing}

A frequency-weighted generalization of the balancing model reduction technique discussed in section 2.2 has been introduced by Enns [2]. The idea is to employ two frequency-dependent weighting matrices, one at the input and the other at the output of the system, before obtaining the balanced realization. Once the balanced realization is obtained, the state truncation method in section 2.2 is applied directly. This approach has the advantage that, by properly choosing the weighting matrices, the reduction error can be made smaller in a particular frequency range while it is allowed to be larger in other frequency ranges.

This method can be applied only to strictly proper systems where there is no direct transfer from the inputs to the outputs, i.e., $D=0$.

For the system represented by

$G(s)=(A, B, C, 0)$,

an input weighting matrix $W_{i}(s)$ having $n_{i}$ states and an output weighting matrix $W_{o}(s)$ having $n_{o}$ states with the realizations

$$
W_{i}(s)=\left(A_{i}, B_{i}, C_{i}, D_{i}\right) \text { and } W_{o}(s)=\left(A_{o}, B_{o}, C_{o}, D_{o}\right)
$$

are chosen. The cascade realization of $G W_{i}(s)$ and $W_{o} G(s)$ are then formed:

$$
\begin{aligned}
& G W_{i}(s)=\left(A_{c}, B_{c}, C_{c}, D_{c}\right) \\
& W_{o} G(s)=\left(A_{o}, B_{o}, C_{o}, D_{o}\right) .
\end{aligned}
$$

The controllability gramian $L_{e}$ of the input-weighted system $G W_{i}(s)$ and the observability gramian $L_{o}$ of the outputweighted system $W_{o} G(s)$ are obtained by solving their defining Lyapunov equations, respectively. Then the frequencyweighted controllability and observability gramians $\hat{L}_{e}$ and $\hat{L}_{o}$ are obtained by extracting the upper left $n \times n$ sub-matrix of $L_{e}$ and $L_{o}$ correspondingly:

$$
L_{e}=\left[\begin{array}{cc}
\hat{L}_{e} & * \\
* & *
\end{array}\right], \quad L_{o}=\left[\begin{array}{cc}
\hat{L}_{0} & * \\
* & *
\end{array}\right] .
$$

The required similarity transformation matrix T is calculated using the eigenvalue-eigenvector decomposition of $L_{e} L_{o}$ as follows:

$$
L_{e} L_{o}=T A T^{-1} \text {. }
$$

Then, the frequency-weighted balanced realization for $\mathrm{G}$ is obtained as

$$
G_{w b}(s)=(\hat{A}, \hat{B}, \hat{C}, 0)=\left(T^{-1} A T, T^{-1} B, C T, 0\right) \text {. }
$$

The model reduction technique discussed in section 2.2 can be applied directly to the realization in (2.18). 
The model reduction error for this case is defined as

$E_{\infty} \equiv\left\|W_{o}(s)\left[G(s)-G_{r}(s)\right] W_{i}(s)\right\|_{\infty}$.

For the case where either $W_{o}=I$ or $W_{i}=I$, the reduced-order model is guaranteed to be stable. However, for the general case of non-unity weighting, no guarantee on the stability can be made.

\subsection{Balanced Truncation with Reduced Error Bound}

This technique embraces both methods described in Section 2.2 and 2.3. In fact, the Balancing Model Reduction and the Low-Frequency Approximation methods fall out to be two special cases of this Balanced Truncation with Reduced Error Bound method proposed by Chow and Rao [1].

Using the common nomenclature given in Section 2.1 equations (2.1) to (2.4), the corresponding system equations are

$$
\begin{aligned}
& \dot{x}_{1}(t)=A_{11} x_{1}(t)+A_{12} x_{2}(t)+B_{1} u(t) \\
& \dot{x}_{2}(t)=A_{21} x_{1}(t)+A_{22} x_{2}(t)+B_{2} u(t) \\
& y(t)=C_{1} x_{1}(t)+C_{2} x_{2}(t)+D u(t) .
\end{aligned}
$$

The states of the weaker sub-system, in complex frequency domain, are approximated as

$$
X_{2}(s) \approx-\delta A_{22}^{-1} A_{21} X_{1}-\delta A_{22}^{-1} B_{2} U(s)
$$

where $\delta$ is a positive real constant and $X_{1}(s), X_{2}(s)$ and $U(s)$ are the Laplace transform of the corresponding state and input vectors. By Laplace transforming equations (2.20) and (2.22) and making the substitution (2.23), the following reduced-order model is obtained:

where

$$
G_{r}(s)=\left(A_{r}, B_{r}, C_{r}, D_{r}\right)
$$

$$
\begin{aligned}
& A_{r}=A_{11}-\delta A_{12} A_{22}^{-1} A_{21} \\
& B_{r}=B_{1}-\delta A_{12} A_{22}^{-1} B_{2} \\
& C_{r}=C_{1}-\delta C_{2} A_{22}^{-1} A_{21} \\
& D_{r}=D-\delta C_{2} A_{22}^{-1} B_{2} .
\end{aligned}
$$

This reduced-order model is unique in terms of input-output behaviour. With the following definitions,

$\bar{A} \equiv A_{12} \sum_{2} A_{22}^{-T} A_{12}^{T}$

and $\quad Q \equiv B_{r} B_{r}^{T}+\delta(\delta-1)\left(\bar{A}+\bar{A}^{T}\right)$

where $\delta$ is a positive real constant, then $G_{r}(s)$ is guaranteed to be stable if and only if $Q$ is positive definite.

By correctly selecting the value of $\delta$, the error bound for this reduced-order model is proven to be lower than those obtained using methods in Sections 2.2 and 2.3. In fact, it is given by

$$
E \leq 2 \sigma_{n} \text {. }
$$

The zero-frequency and infinite-frequency model reduction errors are given by

$$
\begin{aligned}
& E(0)=\lim _{\omega \rightarrow 0} \bar{\sigma}(j \omega)=2 \sigma_{n}\left|\frac{\left(1-\frac{1}{\delta}\right) A_{22}}{-\frac{1}{\delta} A_{22}^{-1}+A_{21} A_{11}^{-1} A_{12}}\right| \\
& E(\infty)=\lim _{\omega \rightarrow \infty} \bar{\sigma}(j \omega)=2 \delta \sigma_{n} .
\end{aligned}
$$

For proofs of the above equations and inequality, please see the theorems and lemmas proposed in Chow [1].

Moore's method corresponds to $\delta=0$ and Prakash's method corresponds to $\delta=1$. For any other values of $\delta$ between 0 and 1, the upper-bound of the model reduction error is lower than that of Moore's and Prakash's. Moreover, the value of $\delta$ can be selected to suite the plant's operating frequency, low or high, so that the model reduction error is made negligible.

Figure 1 below shows the spectral norm of the model reduction error versus frequency for different values of $\delta$. If we choose $\delta=0.5$, the error is about the same throughout the whole frequency range and is equals to one half of the error of the other two methods mentioned.

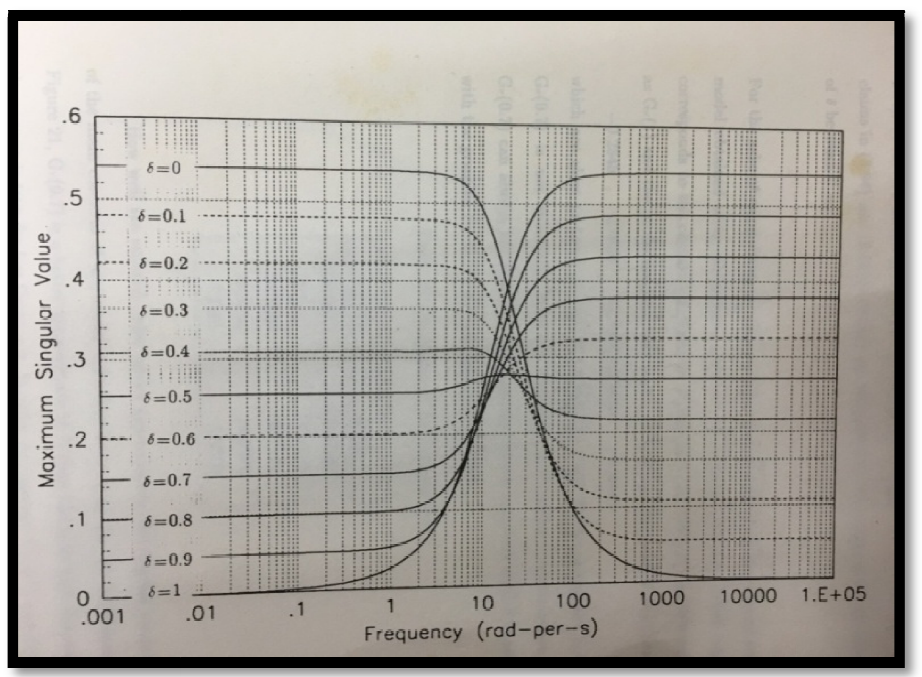

Figure 1 


\section{Numerical Examples}

\subsection{Turbo-Generator}

A $6^{\text {th }}$ order, two-input two-output state-space model for a large turbo-generator taken from [8] is given below.

$$
\begin{aligned}
& A=\left[\begin{array}{cccccc}
-18.4456 & 4.2263 & -2.2830 & 0.2260 & 0.4220 & -0.0951 \\
-4.0977 & -6.0706 & 5.6825 & -0.6966 & -1.2246 & 0.2873 \\
1.4449 & 1.4336 & -2.6477 & 0.6092 & 0.8979 & -0.2300 \\
-0.0093 & 0.2302 & -0.5002 & -0.1764 & -6.3152 & 0.1350 \\
-0.0464 & -0.3489 & 0.7238 & 6.3117 & -0.6886 & 0.3645 \\
-0.0602 & -0.2361 & 0.2300 & 0.0915 & -0.3214 & -0.2087
\end{array}\right] \\
& B=\left[\begin{array}{cc}
-0.2748 & 3.1463 \\
-0.0501 & -9.3737 \\
-0.1550 & 7.4296 \\
0.0716 & -4.9176 \\
-0.0814 & -10.2648 \\
0.0244 & 13.7943
\end{array}\right] \\
& C=\left[\begin{array}{cccccc}
0.5971 & -0.7697 & 4.8850 & 4.8608 & -9.8177 & -8.8610 \\
3.1013 & 9.3422 & -5.6000 & -0.7490 & 2.9974 & 10.5719
\end{array}\right] \\
& D=\left[\begin{array}{ll}
0.00 & 0.00 \\
0.00 & 0.00
\end{array}\right]
\end{aligned}
$$

This model is reduced to a $5^{\text {th }}$ order model using Balanced Model Reduction by Moore [9], Low Frequency Approximation by Prakash [10] and Balanced Truncation with Reduced Error Bound by Chow [1].

The maximum singular value of the original model, marked as 'original', is plotted against frequency as shown in Figure 2.The maximum singular value of the reduced-order model using Balanced Model Reduction method and Low Frequency Approximation method are also plotted in the same graph. As pointed out earlier, Moore's method offers a good match at high frequencies while suffering a large model reduction error at low frequencies. On the other hand, Prakash's method introduces negligible error at low frequencies but has to endure a large discrepancy at high frequencies. As mentioned, these two cases are actually special cases in Chow's method that correspond to $\delta=1$ and $\delta=0$, respectively.

Two reduced-order models were obtained using the method of Balanced Truncation with Reduced Error Bound proposed by Chow, one for $\delta=0.2$ and the other for $=0.7$. The singular values for these two models are also plotted in the same graph in Figure 2for comparison. As shown in the graph, by choosing $\delta=0.2$, we could reduce modelling error at low frequencies compared to Moore's but have to tolerate some errors at high frequencies. On the other hand, by choosing $\delta=0.7$, we tolerate some error at low frequencies compared to Prakash but achieve lower model reduction error at high frequency ranges. If we have chosen $\delta=0.5$, the maximum model reduction error, as shown in Figure 1 above, would be only $50 \%$ of the error compared to the other two said methods throughout the whole frequency range.

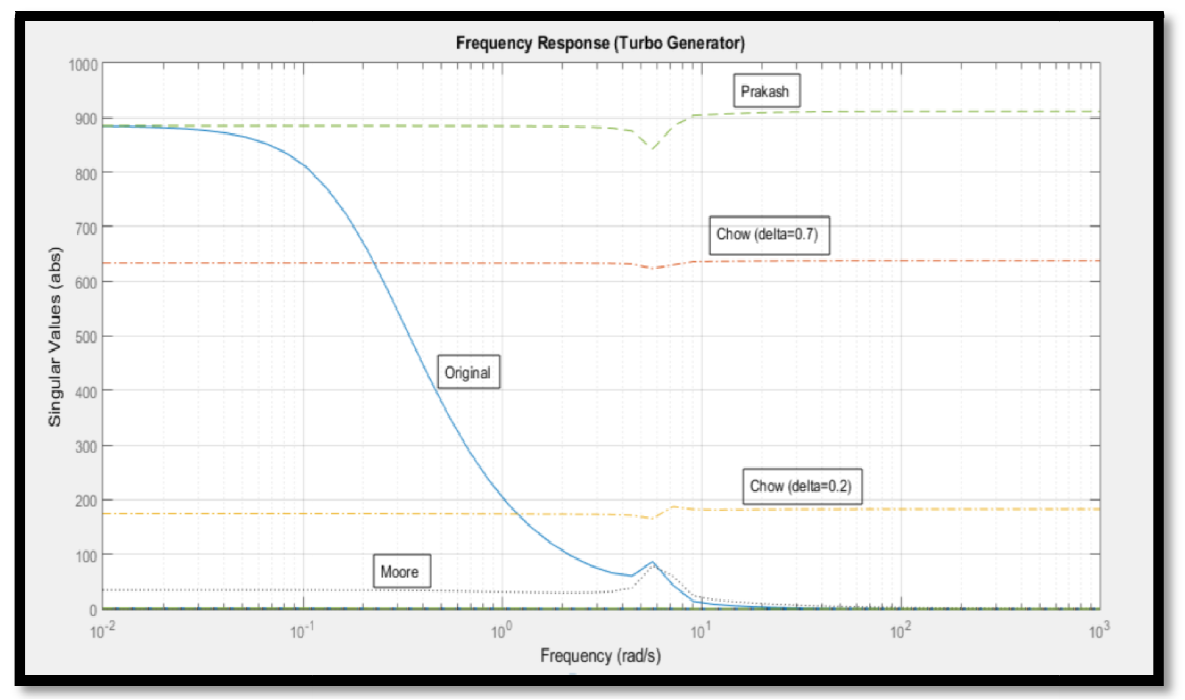

Figure 2

\subsection{Industrial Boiler}

A balanced state-space realization for a large industrial boiler with 8 states, 2 inputs and 2 outputs taken from [5] is given below. 


\begin{tabular}{|c|c|c|c|c|c|c|}
\hline & \multirow{7}{*}{$=\left[\begin{array}{c}0.9963 \\
0.0099 \\
0.0000 \\
0.00 \\
0.00 \\
0.00 \\
0.00 \\
0.00\end{array}\right.$} & 0.00 & 0.00 & 0.00 & 0.000 .00 & 0.00 \\
\hline & & 0.99 & $0.00 \quad 0.0$ & 0.0 & & \\
\hline & & 0.00 & 0.99620 & & & 0.00 \\
\hline & & 0.00 & 0.000 .005 & 0.99 & $0.00 \quad 0.00$ & \\
\hline & & 0.00 & $0.00 \quad 0.00$ & 0.00 & $0.00 \quad 0.00$ & \\
\hline & & $\begin{array}{r}0.00 \\
0.0\end{array}$ & $\begin{array}{cc}0.00 & 0.00 \\
0.000 .00\end{array}$ & $\begin{array}{l}0.00 \\
0.00\end{array}$ & $\begin{array}{l}0.00 \quad 0.00 \\
0.000 .00\end{array}$ & .00 \\
\hline & & & & & 2000 & \\
\hline
\end{tabular}

$$
B=\left[\begin{array}{ll}
0.0037 & 0.0000 \\
0.0000 & 0.0000 \\
0.0000 & 0.0038 \\
0.0000 & 0.0000 \\
0.0030 & 0.0000 \\
0.0000 & 0.0000 \\
0.0000 & 0.0500 \\
0.0000 & 0.0000
\end{array}\right]
$$

$$
\begin{aligned}
C & =\left[\begin{array}{lllllll}
0.00 & 2.20 & 0.00 & 0.033 & 0.00 & 0.000 .00 & 0.00 \\
0.00 & 0.00 & 0.00 & 0.00 & -0.26 & 0.000 .00 & 0.09
\end{array}\right] \\
D & =\left[\begin{array}{ll}
0.00 & 0.00 \\
0.00 & 0.00
\end{array}\right] .
\end{aligned}
$$

This system is selected as a numerical example here due to its unique characteristic that most of its states are so weakly coupled to each other as can be seen in the system matrix, rendering the sub-system to be truncated very insignificant. It turns out that, at high frequencies, all the three methods mentioned here achieve zero model reduction error as shown in Figure 3. (Note that high and low are relative terms.) This reduces to obtaining a reduced order model that has a lower model reduction error at low frequencies, which the method of Prakash prevails.

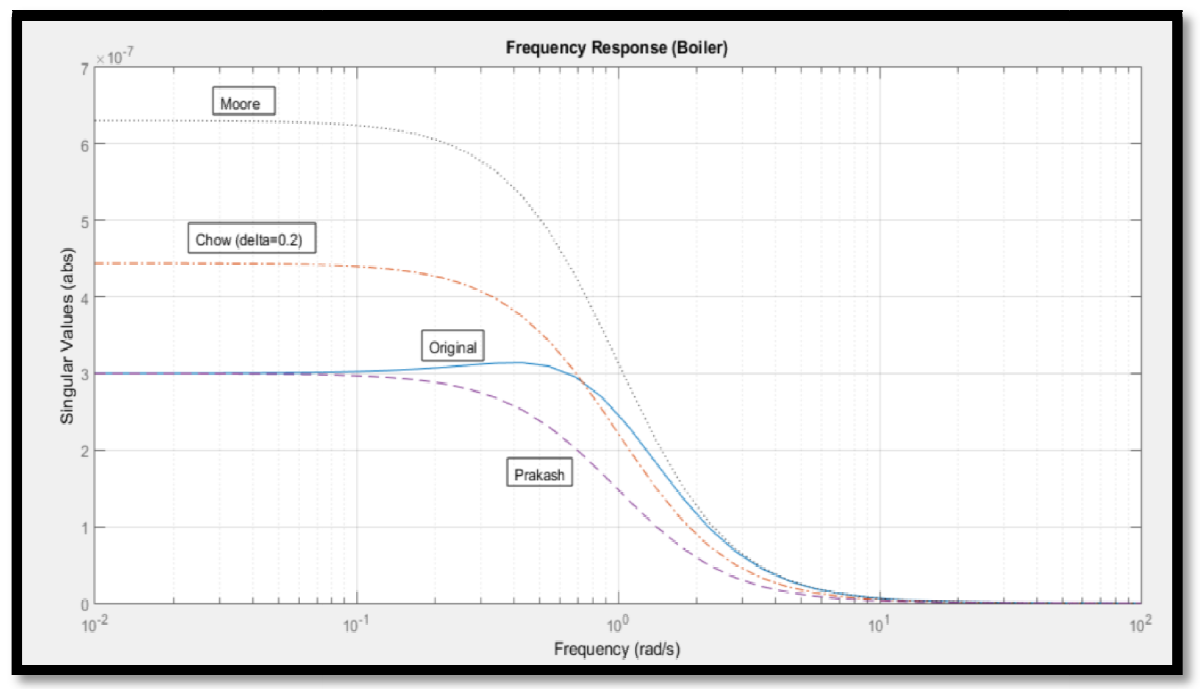

Figure 3

\section{Conclusion}

This paper demonstrates the superiority of the Balanced Truncation with Reduced Error Bound model reduction technique which offer the controller designer an extra degree of freedom in which the spectra norm of the model reduction error at low and high frequencies can be compromised. Specifically, by properly selecting the value of the parameter $\delta$, the relative magnitude of the model reduction error at low and high frequency ranges can be chosen to suit a specific application. This is a welcomed feature because it enables the designer to minimize the model reduction error at a frequency range that most matters for a particular application. If desired, that error could be reduced by half throughout the entire frequency range.

\section{References}

i. Chow S.C. \& Rao V. (1991). Model Reduction Using Balanced-Truncation Method with Lower Error Bounds, 34th Midwest Symposium on Circuit and Systems, Monterey, CA, USA.

ii. Enns D.F. (1984). Model Reduction with Balanced Realizations: An Error Bound and a Frequency Weighted Generalization, Proceedings of the 23 ${ }^{\text {rd }}$ Conference on Decision and Control, Las Vegas, pp.127-132.

iii. Glover K. \& Doyle J. (1988). State-Space Formulae for all Stabilizing Controllers that Satisfy an $H_{\infty}$-norm Bound and Relation to Risk Sensitivity, System and Control Letters, vol. 11, pp.167-172.

iv. Hu X., Stanton, S., Cai, L.\&White R. (2012). Model Order Reduction for Solid-Phase Diffusion in Physics-Based Lithium ion Cell Models, Journal of Power Sources, www.elsevier.com/locate/jpowsour. 
v. Isapour A., Sadati, A.\&Ranjbar A. (2004). Frequency-Domain Stable State-Space System Identification, Transaction of the Institute of Measurement and Control 26-4, pp. 261-272.

vi. Kailath T. (1980). Linear Systems, Prentice-Hall, Englewood Cliffs, New Jersey.

vii. Liu Y.\& Anderson B. (1989). Singular Perturbation Approximation of Balanced Systems, Proceedings of the 28 Conference on Decision and Control, Tampa, pp.1355-1360.

viii. Maciejowski, J. M. (1989). Multivariable Feedback Design, Addison-Wesley, New York.

ix. Moore B.C. (1982). Principle Component Analysis in Linear Systems: Controllability, Observability and Model Reduction, IEEE Transection on Automatic Control, Vol. AC-26, No. 1, pp. 17-32.

x. Prakash R.\& Rao V. (1989). Model Reduction by Low Frequency Approximation of Internally Balanced Representation, Proceedings of the 28 ${ }^{\text {th }}$ Conference on Decision and Control, Tampa, pp.2425-2430.

xi. Ridgely, D.B. (1986). Introduction to Robust Multivariable Control, Lecture notes from Flight Dynamics Laboratory, Ohio.

xii. Zhao D., Yan S., Matsushita S.\& Li X. (2018). An Approach to Multidimensional Fornasini-Marchesini State-Space Model Realization with respect to Columns of Transfer Matrices, Systems \& Control Letters, www.elsevier.com/locate/sysconle. 